Received 11th November 2012, Accepted 8th March 2013

DOI: 10.1039/c3pp25383j

www.rsc.org/pps
View Article Online

View Journal | View Issue

\title{
Modular generation of fluorescent phycobiliproteins $\dagger$
}

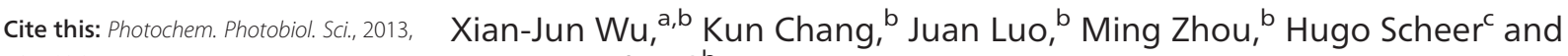 \\ 12, 1036 \\ Kai-Hong Zhao*b
}

Phycobiliproteins are brightly-fluorescent light-harvesting pigments for photosynthesis in cyanobacteria and red algae. They are also of interest as fluorescent biomarkers, but their heterologous generation in vivo has previously required multiple transformations. We report here a modular approach that requires only two DNA segments. The first codes for the apo-protein. The second codes for fusions capable of chromophore biosynthesis and its covalent attachment to the apo-protein; it contains the genes of heme oxygenase, a bilin reductase, and a chromophore lyase. Phycobiliproteins containing phycoerythrobilin $\left(\lambda_{\text {fluor }} \sim 560 \mathrm{~nm}\right)$, phycourobilin $\left(\lambda_{\text {fluor }} \sim 500 \mathrm{~nm}\right)$, phycocyanobilin $\left(\lambda_{\text {fluor }} \sim 630 \mathrm{~nm}\right)$ or phycoviolobilin ( $\lambda_{\text {fluor }} \sim 580 \mathrm{~nm}$ ) were obtained in high yield in E. coli. This approach facilitates chromophorylation studies of phycobiliproteins, as well as their use for fluorescence labeling based on their high fluorescence.

\section{Introduction}

Phycobiliproteins are brightly fluorescing photosynthetic lightharvesting pigments. ${ }^{1-3}$ In cyanobacteria and red algae they are the major components of the large antenna complexes, or phycobilisomes, which consist of a core complex with radiating rods. The rods contain phycocyanin (PC) and often also phycoerythrins (PE) or phycoerythrocyanin (PEC), while the core contains allophycocyanins (APC) and a core-membrane linker. ${ }^{4}$ PEs and PEC absorb between $500-560 \mathrm{~nm}$ and fluoresce at $580 \mathrm{~nm}$, PC absorbs at $615 \mathrm{~nm}$ and fluoresces at $635 \mathrm{~nm}$ (for an example on the absorption and fluorescence properties, see Fig. 1). APC absorbs between $610-650 \mathrm{~nm}$ and fluoresces at $660 \mathrm{~nm}$, while the core-membrane linker absorbs between $610-660 \mathrm{~nm}$ and fluoresces at $670 \mathrm{~nm} .{ }^{5}$ When assembled in the phycobilisome, they form a highly-efficient cascade where excitation energy is transferred from high energy PE or PEC via PC to low energy APC, and eventually is funneled downwards via the core-membrane linker to the reaction centers. ${ }^{1}$ The high fluorescence yield of the biliproteins contributes to the high-efficiency energy transfer, which, in principle, also makes them excellent candidates for fluorescent biomarkers. ${ }^{6-8}$

\footnotetext{
${ }^{a}$ College of Environmental Science and Engineering, Huazhong University of Science and Technology, Wuhan 430074, P. R. China

${ }^{b}$ State Key Laboratory of Agricultural Microbiology, Huazhong Agricultural University, Wuhan 430070, P. R. China. E-mail: khzhao@163.com; Tel: +86-27-87284301

${ }^{c}$ Department Biologie I, Universität München, Menzinger Str. 67, D-80638 München, Germany

$†$ Electronic supplementary information (ESI) available. See DOI: 10.1039/c3pp25383j
}

There are four types of chromophores covalently attached to the phycobiliproteins, namely, phycocyanobilin (PCB), phycoerythrobilin (PEB), phycoviolobilin (PVB) and phycourobilin (PUB). ${ }^{3,9,10}$ All are derived from heme, which is first oxidatively cleaved by heme oxygenase, HO1, to biliverdin, ${ }^{11}$ followed by an enzymatic reduction to PCB through the function of PCB: ferredoxin oxidoreductase, PcyA ${ }^{12}$ if PEB is formed, the PEB: ferredoxin oxidoreductase (PebA/PebB or PebS) is acting instead. ${ }^{13,14}$ Then the chromophores are attached to cysteine residues of their apoproteins by a set of lyases. Some of these lyases are, in addition, capable of isomerizing PCB to PVB, or PEB to PUB. ${ }^{15-18}$

In principle, the strong fluorescence and their variation in excitation and emission wavelengths make phycobiliproteins excellent tools for intracellular applications. The need for chromophore biosynthesis and enzymatic attachment, however, has largely restricted such applications. ${ }^{6}$ It could be shown more recently that cyanobacterial phycobiliproteins have also been assembled heterologously in E. coli. ${ }^{9,10}$ This latter approach required the multi-plasmid-based production of: (i) the apoprotein, (ii) heme oxygenase, (iii) one or more reductases required to generate the chromophore from biliverdin, and (iv) one or more lyase(s). ${ }^{19-21}$ A somewhat simplified approach reducing the number of plasmids required for transformants capable of producing biliproteins has recently been demonstrated. ${ }^{22}$ It is based on expressing the structural gene fused to genes coding for chromophore biosynthesis from endogenous heme. It requires the introduction of only a single gene for labeling and yields fluorescent proteins, which, however, as a disadvantage, exhibit a rather large size due to the fusion approach. ${ }^{22}$ We now report a modular alternative using two sets of plasmids, by this we are able to overcome the 


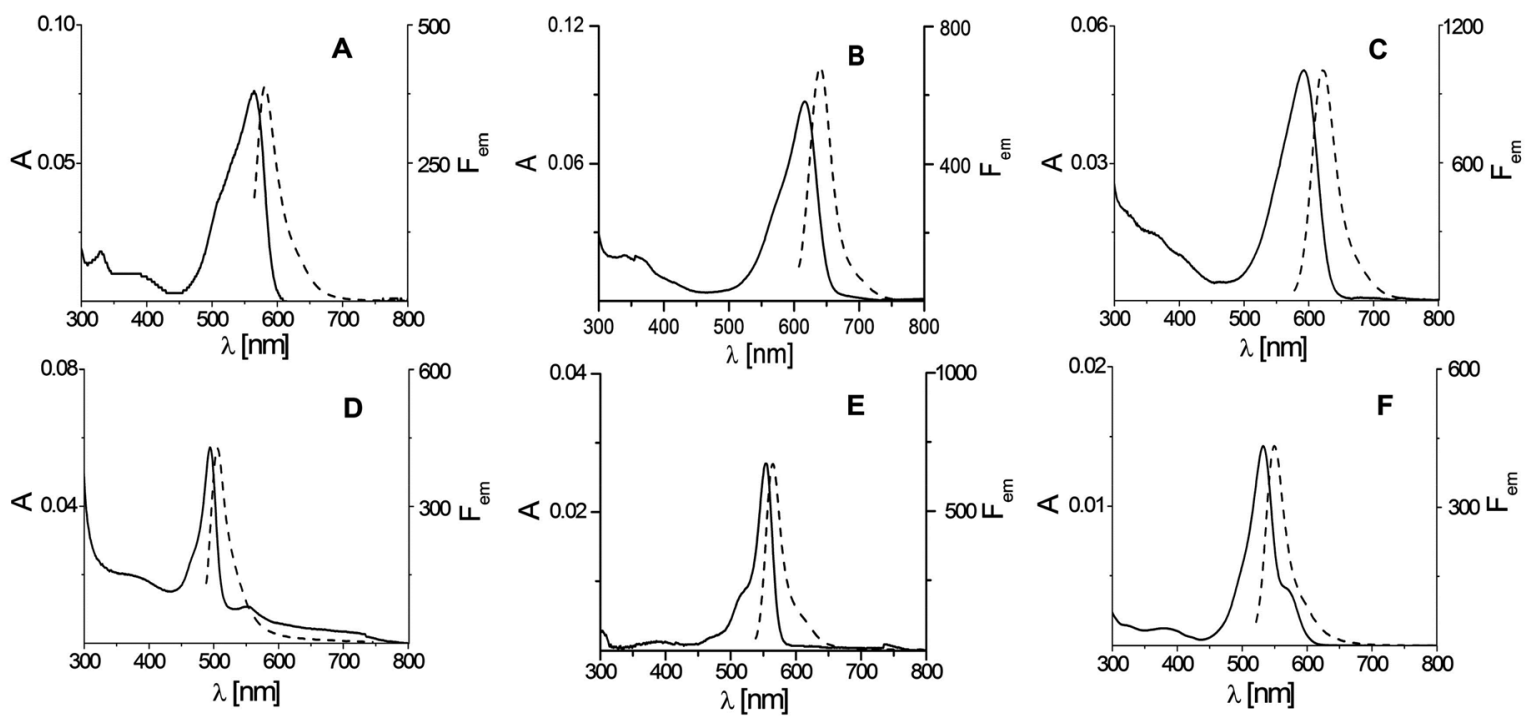

Fig. 1 Absorption (-) and fluorescence emission spectra (---) of PVB-PecA (A), PCB-CpcB(C-84) (B), PCB-CpcB(C-155) (C), PUB-PecA (D), PEB-CpcB(C-84) (E), $\mathrm{PEB}-\mathrm{CpcB}(\mathrm{C}-155)(\mathrm{F})$. The biliproteins were generated by combination of the apoprotein and biosynthesis plasmids shown in Table $\mathrm{S} 2,+$ purified with $\mathrm{Ni}^{2+}$ affinity chromatography and then kept in KPB $(20 \mathrm{mM}, 0.5 \mathrm{M} \mathrm{NaCl}, \mathrm{pH} 7.0)$. Emission spectra were obtained by excitation at $550 \mathrm{~nm}$ for PVB-PecA, $590 \mathrm{~nm}$ for PCB-CpcB (C-84), $560 \mathrm{~nm}$ for PCB-CpcB(C-155), $470 \mathrm{~nm}$ for PUB-PecA, $520 \mathrm{~nm}$ for PEB-CpcB(C-84), and $500 \mathrm{~nm}$ for PEB-CpcB(C-155).

above-mentioned obstacles. Each member of the first set contains a single gene coding for one of the apoproteins. Members of the second set contain, as fusions, all genes required for biosynthesis of a particular chromophore plus its attachment to the desired apoprotein site. By transformation with appropriate pairwise combinations of one member of each set, phycobiliproteins with a molecular weight of $\sim 16 \mathrm{kDa}$ and fluorescing in various colors could be produced in $E$. coli, significantly expanding the toolbox of fluorescent protein tags.

\section{Materials and methods}

\section{Cloning and expression}

All genetic manipulations were carried out according to standard protocols. ${ }^{23}$ In order to construct plasmids for fluorescence labeling of cells in various colors, appropriate gene segments were PCR-amplified from the following previously reported plasmids with the primers listed in the supplement (Table S1 $f$ ): pET-cpcB carrying $c p c B$ that encodes the apoprotein of the $\beta$-subunit of PC $\left(\beta\right.$-CPC); ${ }^{24}$ plasmid pET-pecA carrying pecA that encodes the apo-protein of $\alpha$-PEC of Mastigocladus laminosus $;^{15}$ dual plasmid pACYC-ho1-pcyA carrying ho1 and pcyA of Nostoc sp. PCC7120 (Nostoc) that yields PCB in E. coli; ${ }^{21}$ dual plasmid pACYC-ho1-pebS carrying ho1 and pebS that yields $\mathrm{PEB}$ in $E$. coli; ${ }^{25}$ pCDF-cpcS carrying $c p c S$ that encodes the S-type lyase of Nostoc; ${ }^{26}$ pCDF-cpcT carrying $c p c T$ that encodes the T-type lyase of Nostoc; ${ }^{24}$ and PCDF-rpcG carrying rpcG that encodes RpcG (isomerase-lyase) of Synechococcus sp. WH8102 (Synechococcus). ${ }^{15}$

All PCR products were ligated into the cloning vector pBluescript (Stratagene). After sequence verification, the gene segments were subcloned into the expression vectors pACYC, pCDF or pET30 (Novagen) (Table S2 $\dagger$ ). For various color fluorescence labeling of cells, the pACYC-derived vectors, pCDFderived vectors or pET30-derived vectors were transformed into $E$. coli Tuner ${ }^{\mathrm{TM}}$ (DE3) (Novagen) according to the combinations in Table S2. $†$ The multiply-transformed cells were cultured at $18{ }^{\circ} \mathrm{C}$ in Luria-Bertani (LB) medium supplemented with kanamycin $\left(20 \mu \mathrm{g} \mathrm{ml}^{-1}\right)$, chloromycetin $\left(17 \mu \mathrm{g} \mathrm{ml} \mathrm{m}^{-1}\right)$ or streptomycin $\left(50 \mu \mathrm{g} \mathrm{ml^{-1 }}\right)$. We adjusted the growth temperature and expression time after induction of cells, instead of the concentration of isopropyl $\beta$-D-thiogalactoside, which lets phycobiliproteins be assembled gradually and cells be labeled well. After induction with isopropyl $\beta$-D-thiogalactoside $(1 \mathrm{mM})$ for 4-12 hours, the cells were centrifuged at $12000 \mathrm{~g}$ for 3 minutes at $4{ }^{\circ} \mathrm{C}$.

\section{Protein assay}

For isolation of the fluorescent proteins, the cell pellet was resuspended in ice-cold potassium phosphate buffer (KPB, $20 \mathrm{mM}, \mathrm{pH} 7.0$ ) containing $0.5 \mathrm{M} \mathrm{NaCl}$ and disrupted by sonication for $5 \mathrm{~min}$ at $200 \mathrm{~W}$ (JY92-II, Scientz Biotechnology, Ningbo, China). The suspension was centrifuged at $12000 \mathrm{~g}$ for $15 \mathrm{~min}$ at $4{ }^{\circ} \mathrm{C}$ and the supernatant purified via $\mathrm{Ni}^{2+}$-affinity chromatography on chelating Sepharose (Amersham Biosciences) developed with KPB containing $0.5 \mathrm{M} \mathrm{NaCl}$. The bound proteins remaining on the column were eluted with the saline KPB, containing in addition imidazole (0.5 M). After collection, the protein sample was dialyzed twice against the saline KPB. ${ }^{21}$

Protein concentrations were determined by the Bradford assay, ${ }^{27}$ calibrated with bovine serum albumin and SDS-PAGE was performed using the buffer system of Laemmli. ${ }^{28}$ Proteins 
were stained with Coomassie brilliant blue and those containing bilins were identified by $\mathrm{Zn}^{2+}$-induced fluorescence. ${ }^{29}$

\section{Spectral analyses}

All chromoproteins were investigated by UV-Vis absorption spectroscopy (Beckman-Coulter DU 800). Covalently bound PCB, PEB, PUB or PVB in biliprotein derivatives were quantified after denaturation with acidic urea $(8 \mathrm{M}, \mathrm{pH} 1.5)$ by their absorption at: $662 \mathrm{~nm}\left(\varepsilon=35500 \mathrm{M}^{-1} \mathrm{~cm}^{-1}\right)$ for $\mathrm{PCB}{ }^{30}$ $550 \mathrm{~nm}\left(\varepsilon=42800 \mathrm{M}^{-1} \mathrm{~cm}^{-1}\right)$ for PEB; ${ }^{31} 495 \mathrm{~nm}(\varepsilon=104000$ $\left.\mathrm{M}^{-1} \mathrm{~cm}^{-1}\right)$ for PUB; ${ }^{32}$ and, $595 \mathrm{~nm}\left(\varepsilon=38600 \mathrm{M}^{-1} \mathrm{~cm}^{-1}\right)$ for PVB. ${ }^{33}$ Fluorescence spectra were recorded at room temperature with a model LS 55 spectrofluorimeter (Perkin-Elmer). Fluorescence quantum yields, $\Phi_{\mathrm{F}}$, were determined in KPB ( $\mathrm{pH}$ 7.0) using the known $\Phi_{\mathrm{F}}=0.27$ of C-PC from Nostoc ${ }^{34}$ and $\Phi_{\mathrm{F}}=0.98$ of the biosynthetically-obtained $\mathrm{PEB}-\mathrm{CpcA}^{35}$ as standards.

\section{Microscopic analysis}

The appropriate strains were induced to express the required phycobiliprotein label and grown for several hours, until the fluorescence became visible by eye. After harvesting, the cells were deposited on a glass slide, and micrographs taken with a fluorescence microscope (Immersol 518F, Carl Zeiss) fitted with a color CCD camera (SPOT RT3 25.2 2 Mp color Mosaic, SPOT).

\section{Results and discussion}

Fusions of phycobiliprotein lyases, HO1 and PcyA (or PebS) have the activities of both lyases and phycobilin biosynthesis

It was shown earlier that the heterologous biosynthesis of biliproteins can be simplified by using fusion proteins; an example is the fusion of HO1::PcyA which produces PCB in E. coli. ${ }^{22}$ This approach has now been extended by adding a lyase gene, $r p c G, c p c S$ or $c p c T$, to the plasmid containing ho1::pcyA. The resulting plasmids in $E$. coli generated the following fusion proteins: RpcG::HO1::PcyA; CpcS::HO1::PcyA; and CpcT::HO1::PcyA (Table S2†). The first product contains the $\mathrm{E} / \mathrm{F}$ type of isomerase-lyase, RpcG in which the $\mathrm{E}$ and $\mathrm{F}$ subunits are already fused; it can isomerize PCB to PVB and PEB to PUB, and then simultaneously attach the isomerized bilins to suitable apo-proteins. ${ }^{15}$ All three activities of the original enzyme components were still active in the fusion product, as shown by co-expression with appropriate apoproteins yielding orange-fluorescent proteins carrying the PVB chromophore (Fig. 1A). Thus, only two, instead of four, DNA segments have to be introduced to generate fluorescent holoproteins (Table S2†). Similarly, red-fluorescent proteins were generated by constructs combining the PCB generation (HO1, PcyA) with the non-isomerizing S- or T-type lyases (Fig. 1B and C). S-type lyases catalyze the covalent attachment of PCB at C-84 of both $\beta$-CPC and $\beta$-PEC, and at many other related sites of both subunits of APC and PE. T-type lyases are specific for the C-155 site of both $\beta$-CPC and $\beta$-PEC. ${ }^{24,26,36}$

In order to expand the spectral variation of fluorescent biliprotein labels, biliverdin has been attached to the variants derived from bacterial phytochrome. ${ }^{37,38}$ However, neither phycobiliproteins nor phycobiliprotein lyases could accommodate biliverdin, ${ }^{9}$ so PEB was tried in this work. PEB can be synthesized from biliverdin by the heterodimeric PebA/PebB lyase $;^{12}$ the single-subunit lyase, PebS, combines the activities of both subunits. ${ }^{13}$ We therefore chose pebs for PEB production and generated the following constructs: $r p c G:: h o 1::$ pebS; cpcS::ho1::pebS; and, cpcT::ho1::pebS (Table S2†). RpcG:: HO1::PebS had both isomerase-lyase activity from RpcG and PEB-producing activity from HO1::PebS. Together with a suitable apo-protein such as, for example, PecA (apo- $\alpha$-phycoerythrocyanin), bluish-green fluorescing phycobiliproteins containing PUB can be obtained (Fig. 1D). ${ }^{15}$ CpcS::HO1::PebS or CpcT::HO1::PebS combined S-lyase or T-lyase activity with PEB-producing activity. Together with suitable apo-proteins such as, for example, CpcB (apo- $\beta$-phycocyanin), this resulted in orange fluorescing phycobiliproteins containing PEB (Fig. $1 \mathrm{E}$ and $\mathrm{F}$ ). ${ }^{15}$

When RpcG or CpcT were fused with either HO1::PcyA or HO1::PebS, the relative activities were lower ( $~ 50 \%)$ compared with the formerly reported four-plasmid approach (Table 1$) ;^{15,21}$ however, when the fusion constructs contained $c p c S$, the holoprotein yield was actually increased compared with the four-plasmid approach. When $\mathrm{CpcS}$ was fused with

Table 1 Quantitative absorption and fluorescence data of the fused phycobiliproteins

\begin{tabular}{|c|c|c|c|c|c|c|}
\hline \multirow[b]{2}{*}{ Fused phycobiliproteins } & \multicolumn{2}{|c|}{ Absorption } & \multicolumn{2}{|c|}{ Fluorescence } & \multirow[b]{2}{*}{ Fused enzymes } & \multirow[b]{2}{*}{ Relative $^{a}$ activity } \\
\hline & $\lambda_{\max }[\mathrm{nm}]$ & $\varepsilon_{\text {Vis }}\left[\mathrm{M}^{-1} \mathrm{~cm}^{-1}\right]$ & $\lambda_{\max }[\mathrm{nm}]$ & $\Phi_{\mathrm{F}}$ & & \\
\hline PVB-PecA & 564 & $9.6( \pm 0.2) \times 10^{4}$ & 582 & $0.07( \pm 0.01)$ & RpcG::HO1::PcyA & $40 \%$ \\
\hline PUB-PecA & 494 & $15.2( \pm 0.3) \times 10^{4}$ & 506 & $0.25( \pm 0.01)$ & RpcG::HO1::PebS & $58 \%$ \\
\hline PCB-CpcB(C-84) & 616 & $12.9( \pm 0.4) \times 10^{4}$ & 641 & $0.16( \pm 0.01)$ & CpcS::HO1::PcyA & $132 \%$ \\
\hline PEB-CpcB(C-84) & 553 & $13.7( \pm 0.2) \times 10^{4}$ & 566 & $0.80( \pm 0.02)$ & CpcS::HO1::PebS & $265 \%$ \\
\hline PCB-CpcB(C-155) & 592 & $11.1( \pm 0.5) \times 10^{4}$ & 621 & $0.49( \pm 0.02)$ & СрсT::HO1::PcyA & $58 \%$ \\
\hline PEB-CpcB(C-155) & 531 & $8.7( \pm 0.5) \times 10^{4}$ & 551 & $0.99( \pm 0.02)$ & СpcT::HO1::PebS & $66 \%$ \\
\hline
\end{tabular}

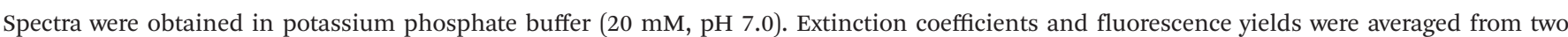

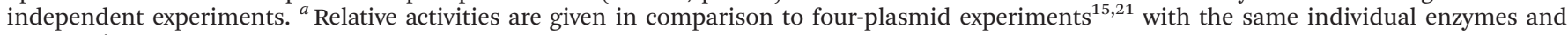
apoproteins. 
HO1::PcyA and, especially, with HO1::PebS, the relative activities were markedly enhanced (see Table 1): the effect is particularly striking (160\% enhancement) with CpcS::HO1::PebS. CpcS catalyzes the chromophorylation of phycobiliproteins by a chaperone-like process. ${ }^{39}$ It first binds phycobilin in an appropriate conformation, and then shuttles phycobilin to the apo-phycobiliprotein. The fusion may facilitate the shuttling process. Lower yields can have various reasons, including, in particular, conformational changes caused by the fusion.

\section{The fusions can be applied in various color fluorescence labeling}

The activity of fusions containing lyases and phycobilin-yielding enzymes (Table 1) considerably simplifies the biosynthesis of phycobiliproteins: in their presence, apo-phycobiliproteins are smoothly chromophorylated (Fig. 1). The resulting singlychromophorylated phycobiliproteins fluoresce in the green, yellow/orange or red spectral regions, depending on the presence of PUB, PEB/PVB or PCB, respectively, as chromophore (Fig. 2). The well-separated narrow excitation and fluorescence emission bands are advantageous for multi-color labeling (Fig. 2). A combination of PEB as donor with PCB as acceptor is difficult because most lyases and apoproteins do not discriminate these chromophores. A better approach for two-color labeling is by combining a non-modifying apoprotein with a modifying apoprotein. This is similar to the biosynthesis of phycoerythrocyanin, where PCB is attached unmodified to the $\beta$-subunit, while being transformed to PVB in the $\alpha$-subunit; this provides for excellent FRET in cyanobacterial light-harvesting. Using PecA with $\mathrm{CpcB}(\mathrm{C}-84)$ in combination with $\mathrm{PCB}$, the absorption maxima are at 564 and $616 \mathrm{~nm}$, the fluorescence maxima at 582 and $641 \mathrm{~nm}$; combining them instead with PEB, the absorption maxima are at 494 and $553 \mathrm{~nm}$, and the
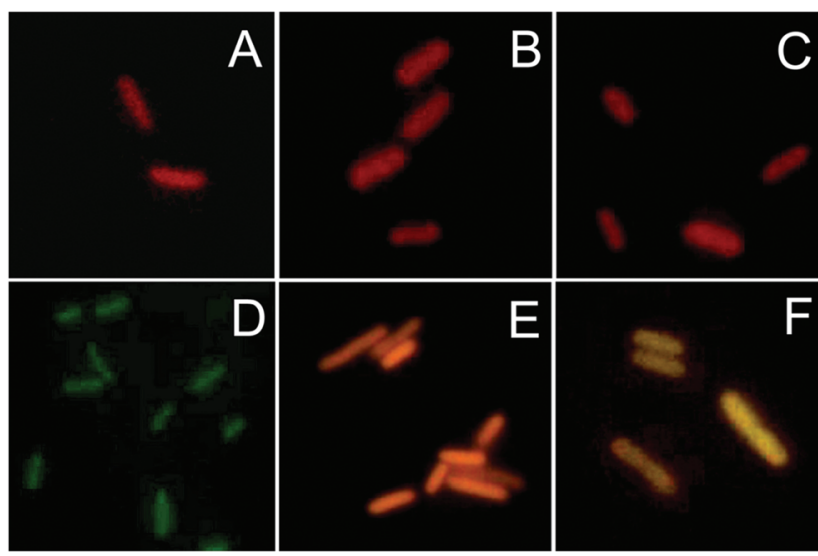

Fig. 2 In situ fluorescence of phycobiliproteins generated in $E$. coli cells by the two-plasmid approach. Fluorescence micrographs of PVB-PecA (A), PCB-CpcB$(C-84)(B), P C B-C p c B(C-155)(C), P U B-P e c A(D), P E B-C p c B(C-84)(E)$, and PEB-CpcB$(C-155)(F)$. The biliproteins were generated by combination of the apoprotein and biosynthesis plasmids shown in Table S2.t Fluorescence measurements were done with an excitation filter (540-552 nm) and a long-pass fluorescence filter $(\geq 590 \mathrm{~nm}$ ) for chromoproteins containing PCB or PVB, and with an excitation filter (450-490 nm) and a long-pass fluorescence filter ( $\geq 515 \mathrm{~nm}$ ) for chromoproteins containing PEB or PUB. fluorescence maxima at 506 and $566 \mathrm{~nm}$ (Table 1). These are good separations for FRET experiments, they can even be enlarged if the modifying apoprotein is generating a urobilin chromophore as donor (absorption at $430 \mathrm{~nm}$ ).

The results with the three lyases in this study indicate that the same approach may be extended to other apoproteins and lyases. The two-plasmid approach principally allows any combination of chromophore with a suitable apoprotein. The approach is restricted by the specificity of some of the lyases, but the known matched combinations can generate biliproteins that cover practically continuously the spectroscopic region from 500-670 $\mathrm{nm} .^{9,10,16,40,41}$ On multi-color labeling, auto-catalytic biliproteins that can be chromophorylated in the absence of lyases, such as the chromophore domains of phycobilisome core-membrane linker and/or cyanobacteriochromes, ${ }^{22,25}$ could be applied. Since the biliproteins are autocatalytically chromophorylated, we do not need more lyases for multi-color labeling, so simplifying the construction of plasmids. The approach is also advantageous compared with previously used single-plasmid approaches that generated rather large chromophore-containing fusion proteins. ${ }^{22}$ Instead, the approach now generates biliproteins of only $\sim 150$ amino acids, which is an essential pre-condition for applications as protein tags in living cells. It also allows rapid screening for further downsizing, and seems at the same time useful for studying not only phycobiliprotein biosynthesis but also phycobilisome assembly, besides many imaginable applications in studying intracellular protein-protein interactions using modern microscopic techniques.

\section{Abbreviations}

\section{APC \\ HO1 \\ KPB \\ Nostoc \\ PC \\ PCB \\ PcyA \\ PE \\ PEB \\ PEC \\ PebS \\ PVB \\ RpcG}

Synechococcus

Allophycocyanin

Heme oxygenase 1

Potassium phosphate buffer

Anabaena (Nostoc) sp. PCC 7120

Phycocyanin (prefixes stand for C: cyanobacteria, R: rhodophyte)

Phycocyanobilin

PCB: ferredoxin oxidoreductase

Phycoerythrin

Phycoerythrobilin

Phycoerythrocyanin

PEB synthase

Phycoviolobilin

Phycourobilin: $\alpha$-R-PC isomerase-lyase of Synechococcus

\section{Acknowledgements}

We are grateful to R. J. Porra (CSIRO-Plant Industry, Canberra, Australia) for help in preparing the manuscript. HS, KHZ (grant 31110103912) and KHZ (grant 21072068) are grateful for support by the National Natural Science Foundation of China. 


\section{References}

1 E. Gantt, in Photosynthesis III: Photosynthetic Membranes and Light-Harvesting Systems, ed. L. A. Staehelin and C. J. Arntzen, Springer Verlag, Berlin, 1986, pp. 260-268.

2 A. N. Glazer, Biochim. Biophys. Acta, Rev. Bioenerg., 1984, 768, 29-51.

3 W. A. Sidler, in The molecular biology of cyanobacteria, ed. D. A. Bryant, Kluwer, Dordrecht, 1994, pp. 139-216.

4 A. N. Glazer, Adv. Mol. Cell Biol., 1994, 10, 119-149.

$5 \mathrm{H}$. Scheer, in Light Reaction Path of Photosynthesis, ed. F. K. Fong, Springer, Berlin, 1982, pp. 7-45.

6 A. Glazer, J. Appl. Phycol., 1994, 6, 105-112.

7 A. N. Glazer and L. Stryer, Biophys. J., 1983, 43, 383-386.

8 V. T. Oi, A. N. Glazer and L. Stryer, J. Cell Biol., 1982, 93, 981-986.

9 H. Scheer and K. H. Zhao, Mol. Microbiol., 2008, 68, 263-276.

10 W. M. Schluchter, G. Shen, R. M. Alvey, A. Biswas, N. A. Saunee, S. R. Williams, C. A. Mille and D. A. Bryant, Adv. Exp. Med. Biol., 2010, 675, 211-228.

11 S. I. Beale, Chem. Rev., 1993, 93, 785-802.

12 N. Frankenberg and J. C. Lagarias, in The Porphyrin Handbook, ed. K. M. Kadish, K. M. Smith and R. Guilard, Academic Press, Amsterdam, 2003, pp. 211-236.

13 T. Dammeyer, S. C. Bagby, M. B. Sullivan, S. W. Chisholm and N. Frankenberg-Dinkel, Curr. Biol., 2008, 18, 442-448.

14 T. Dammeyer and N. Frankenberg-Dinkel, J. Biol. Chem., 2006, 281, 27081-27089.

15 N. Blot, X. J. Wu, J. C. Thomas, J. Zhang, L. Garczarek, S. Böhm, J. M. Tu, M. Zhou, M. Plöscher, L. Eichacker, F. Partensky, H. Scheer and K. H. Zhao, J. Biol. Chem., 2009, 284, 9290-9298.

16 A. Shukla, A. Biswas, N. Blot, N. F. Partensky, J. A. Karty, L. A. Hammad, L. Garczarek, A. Gutu, W. M. Schluchter and D. M. Kehoe, Proc. Natl. Acad. Sci. U. S. A., 2012, 109, 20136-20141.

17 M. Storf, A. Parbel, M. Meyer, B. Strohmann, H. Scheer, M. G. Deng, M. Zheng, M. Zhou and K. H. Zhao, Biochemistry, 2001, 40, 12444-12456.

18 K. H. Zhao, M. G. Deng, M. Zheng, M. Zhou, A. Parbel, M. Storf, M. Meyer, B. Strohmann and H. Scheer, FEBS Lett., 2000, 469, 9-13.

19 N. A. Saunee, S. R. Williams, D. A. Bryant and W. M. Schluchter, J. Biol. Chem., 2008, 283, 7513-7522.

20 A. J. Tooley, Y. A. Cai and A. N. Glazer, Proc. Natl. Acad. Sci. U. S. A., 2001, 98, 10560-10565.
21 K. H. Zhao, P. Su, J. M. Tu, X. Wang, H. Liu, M. Plöscher, L. Eichacker, B. Yang, M. Zhou and H. Scheer, Proc. Natl. Acad. Sci. U. S. A., 2007, 104, 14300-14305.

22 J. Zhang, X. J. Wu, Z. B. Wang, Y. Chen, X. Wang, M. Zhou, H. Scheer and K. H. Zhao, Angew. Chem., Int. Ed., 2010, 49, 5456-5458.

23 J. Sambrook, E. Fritsch and T. Maniatis, Molecular cloning: a laboratory manual, Cold Spring Harbour Laboratory Press, New York, 1989.

24 K. H. Zhao, J. Zhang, J. M. Tu, S. Böhm, M. Plöscher, L. Eichacker, C. Bubenzer, H. Scheer, X. Wang and M. Zhou, J. Biol. Chem., 2007, 282, 34093-34103.

25 K. Tang, X. L. Zeng, Y. Yang, Z. B. Wang, X. J. Wu, M. Zhou, D. Noy, H. Scheer and K. H. Zhao, Biochim. Biophys. Acta, Bioenerg., 2012, 1817, 1030-1036.

26 K. H. Zhao, P. Su, J. Li, J. M. Tu, M. Zhou, C. Bubenzer and H. Scheer, J. Biol. Chem., 2006, 281, 8573-8581.

27 M. Bradford, Anal. Biochem., 1976, 72, 248-254.

28 U. Laemmli, Nature, 1970, 227, 680-685.

29 T. Berkelman and J. C. Lagarias, Anal. Biochem., 1986, 156, 194-201.

30 A. N. Glazer and S. Fang, J. Biol. Chem., 1973, 248, 659-662.

31 A. N. Glazer and C. S. Hixson, J. Biol. Chem., 1975, 250, 5487-5495.

32 A. N. Glazer and C. S. Hixson, J. Biol. Chem., 1977, 252, 32-42.

33 J. E. Bishop, H. Rapoport, A. V. Klotz, C. F. Chan, A. N. Glazer, P. Füglistaller and H. Zuber, J. Am. Chem. Soc., 1987, 109, 875-881.

34 Y. A. Cai, J. T. Murphy, G. J. Wedemayer and A. N. Glazer, Anal. Biochem., 2001, 290, 186-204.

35 R. M. Alvey, A. Biswas, W. M. Schluchter and D. A. Bryant, Biochemistry, 2011, 50, 4890-4902.

36 G. Shen, N. A. Saunee, S. R. Williams, E. F. Gallo, W. M. Schluchter and D. A. Bryant, J. Biol. Chem., 2006, 281, 17768-17778.

37 G. S. Filonov, K. D. Piatkevich, L. M. Ting, J. Zhang, K. Kim and V. V. Verkhusha, Nat. Biotechnol., 2011, 29, 757-761.

38 X. Shu, A. Royant, M. Z. Lin, T. A. Aguilera, V. Lev-Ram, P. A. Steinbach and R. Y. Tsien, Science, 2009, 324, 804-807.

39 M. Kupka, J. Zhang, W. L. Fu, J. M. Tu, S. Böhm, P. Su, Y. Chen, M. Zhou, H. Scheer and K. H. Zhao, J. Biol. Chem., 2009, 284, 36405-36414.

40 A. Biswas, M. N. Boutaghou, R. M. Alvey, C. M. Kronfel, R. B. Cole, D. A. Bryant and W. M. Schluchter, J. Biol. Chem., 2011, 286, 35509-35521.

41 J. Wiethaus, A. W. Busch, K. Kock, L. I. Leichert, C. Herrmann and N. Frankenberg-Dinkel, J. Biol. Chem., 2010, 285, 37561-37569. 